\title{
Many-body vertex corrections in a one-dimensional electron system interacting with a long-range Coulomb potential
}

\author{
B. Tanatar and E. Demirel \\ Department of Physics, Bilkent University, Bilkent, 06533 Ankara, Turkey
}

(Received 16 February 2000)

\begin{abstract}
We study the quasiparticle properties of a one-dimensional electron gas interacting with a long-range electron-electron interaction. The electron self-energy is calculated using the leading-order dynamicalscreening approximation with ( $G W \Gamma$ approximation) and without the vertex corrections ( $G W$ approximation). The calculated one-electron properties such as the spectral function, damping rate, and also the momentum distribution indicate the significance of vertex corrections at low densities.
\end{abstract}

\section{INTRODUCTION}

Models of one-dimensional (1D) electron systems ${ }^{1}$ are of increasing interest because of their applicability to realistic systems such as naturally occurring organic conductors, artificially fabricated semiconductor structures, and certain materials exhibiting superconductivity. Quantum-wire structures made out of semiconducting materials using highly developed processing techniques provide a testing ground for the many-body theories describing the dynamics of interacting electrons in restricted geometries. A one-dimensional system of electrons interacting via a long-range Coulomb potential in configuration space is a model being used to understand various properties of many realistic systems.

In this work we study the quasiparticle properties of a 1D electron system interacting with a long-range Coulomb potential. We calculate the wave vector and frequencydependent self-energy within the $G W$ and $G W \Gamma$ approximations from which all one-electron properties can be obtained. There are several motivations for our investigation. First, we explore the extent the Fermi liquid theory can be employed in the description of this model system. It has been shown by $\mathrm{Hu}$ and Das Sarma ${ }^{2}$ that disorder and finite-temperature effects render the Fermi liquid picture meaningful in 1D electron gas, and we adopt this viewpoint with application to semiconducting quantum wires in mind. Numerous studies $^{3-7}$ were devoted to the ground-state energy and correlation functions of quasi-one-dimensional electron gas interacting via long-range Coulomb interaction, but relatively less attention is paid to the quasiparticle properties of these models. ${ }^{2,8}$ On the other hand, models of 1D interacting electrons on a lattice are actively being pursued to understand various phenomena, most notable being quantum phase transitions. Second, we investigate the effects of vertex corrections. The extension of the random-phase approximation (RPA) $G W$ approach is formulated by the $G W \Gamma$ approximation, where $\Gamma$ stands for the vertex corrections. In the present model we use the previously obtained ${ }^{4}$ local-field factors to describe the vertex corrections and assess their importance in the quasiparticle properties at low densities.

Within the Tomonaga-Luttinger model of 1D electrons having a linear dispersion relation, the RPA is argued to be exact because the vertex corrections to the irreducible polar- izability vanish. ${ }^{9}$ This fact has been used to explain the agreement between the experimentally observed ${ }^{10}$ plasmon dispersions in quantum wires and the model calculations. ${ }^{11}$ In a system with long-range Coulomb interactions and quadratic energy dispersion, the vertex corrections are not required to vanish identically, rather they should become more significant as the interaction strength between the electrons is increased. The evidence for this comes from the approximate ground-state energy calculations ${ }^{3-7}$ in comparison to the RPA, and also from the recent quantum Monte Carlo simulations. ${ }^{12}$ Thus, it seems reasonable to calculate the quasiparticle properties including the vertex corrections that are consistent with the ground-state energy calculations, similar to the analysis given for $2 \mathrm{D}$ systems. ${ }^{13,14} \mathrm{We}$ use the $G W \Gamma$ approximation in the calculation of electron self-energy where the vertex function is approximated by the local-field factors calculated in the self-consistent-field approximation.

The rest of this paper is organized as follows. In the next section we provide the theoretical background for $G W$ and $G W \Gamma$ approximations to the electron self-energy. In Sec. III we present our numerical results of the self-energy and related quantities calculated from it. We conclude with a brief summary of our results.

\section{MODEL AND THEORY}

We consider a system of electrons in 1D interacting with a long-range Coulomb potential, embedded in a uniform background of positive charges insuring overall charge neutrality. The bare interaction potential $V(q)$ is modeled as obtained from the zero-thickness 2D electron gas under a harmonic confining potential. ${ }^{15}$ This yields $V(q)$ $=\left(e^{2} / \epsilon_{0}\right) e^{x} K_{0}(x)$ for the interaction between the electrons assumed to be in the lowest subband. Here $x=(b q / 2)^{2}$, where $b$ is the lateral width of the quantum wire determined by the confining oscillator frequency and $\epsilon_{0}$ is the background dielectric constant. The system is characterized by the dimensionless density parameter $r_{s}=a / a_{B}^{*}$, where $a$ is the average interparticle distance (i.e., $n=1 / 2 a$ in terms of the linear number density $n)$ and $a_{B}^{*}=\epsilon_{0} /\left(m^{*} e^{2}\right)$ is the effective Bohr radius (we take $\hbar=1$ ). The single-subband approximation, which implies that the Fermi energy remains smaller than the intersubband energy difference, is justified 
for $r_{s}>\left(\pi / 2^{5 / 2}\right)\left(b / a_{B}^{*}\right)$. The Fermi wave vector is related to the linear density by $n=2 k_{F} / \pi$, in an unpolarized system.

The self-energy of the one-dimensional (1D) electron system within the $G W$ approximation (excluding the vertex corrections) at $T=0$ is given by ${ }^{16}$

$$
\Sigma(k, \omega)=i \int \frac{d q d \omega^{\prime}}{(2 \pi)^{2}} W\left(q, \omega^{\prime}\right) G_{0}\left(k-q, \omega-\omega^{\prime}\right),
$$

where $G_{0}(k, \omega)$ is the Green's function for the noninteracting electron gas, with single-particle energies $\xi_{k}=k^{2} / 2 m$ $-\mu$ ( $\mu$ is the chemical potential or the Fermi energy $E_{F}$ $=k_{F}^{2} / 2 m$ at $\left.T=0\right)$. $W(q, \omega)$ is the dynamically screened interaction, which is given by $W(q, \omega)=V(q) / \varepsilon(q, \omega)$. In the effective interaction above $\varepsilon(q, \omega)$ is the dielectric function describing the dynamical screening properties of the electron gas. We employ the usual practice of separating the dynamically screened interaction $W(q, \omega)$ into a frequencyindependent term which gives the exchange part of the selfenergy and a frequency-dependent term which gives the correlation part of the self-energy $W(q, \omega)=V(q)+V(q)$ $\times[1 / \varepsilon(q, \omega)-1]$. The exchange part is given by $\Sigma_{\mathrm{ex}}(k)=$ $-\int_{-\infty}^{\infty}(d q / 2 \pi) n_{F}(k+q) V(q)$, where $n_{F}(k)=\theta\left(k_{F}-k\right)$ is the Fermi distribution function at $T=0$. In the $G W$ approximation, the correlation part of the self-energy can be decomposed into two parts, ${ }^{16} \Sigma_{\text {cor }}(k, \omega)=\Sigma_{\text {line }}(k, \omega)+\Sigma_{\text {pole }}(k, \omega)$. Since $\varepsilon(q, i \omega)$ is a real and even function with respect to $\omega$, the $\Sigma_{\text {line }}(k, \omega)$ term is completely real. The self-energy calculation which includes vertex corrections is called the $G W \Gamma$ approximation. ${ }^{17}$ The importance of vertex corrections in strongly correlated systems was recently emphasized in a number of works. ${ }^{18}$ In the $G W \Gamma$ approximation, $\Sigma_{\text {cor }}(k)$ is again split into two parts, $\Sigma_{\text {line }}(k, \omega)$ and $\Sigma_{\text {pole }}(k, \omega)$, which are given, respectively, by

$$
\begin{aligned}
\Sigma_{\text {line }}(k, \omega)= & -\int_{-\infty}^{\infty} \frac{d \omega^{\prime}}{2 \pi} \int_{-\infty}^{\infty} \frac{d q}{2 \pi} V(q) \\
& \times \frac{1}{\left(\xi_{k+q}-\omega\right)-i \omega^{\prime}}\left[\frac{\Gamma\left(q, i \omega^{\prime}\right)}{\varepsilon\left(q, i \omega^{\prime}\right)}-1\right]
\end{aligned}
$$

and

$$
\begin{aligned}
\Sigma_{\text {pole }}(k, \omega)= & \int_{-\infty}^{\infty} \frac{d q}{2 \pi} V(q)\left[\frac{\Gamma\left(q, \xi_{k+q}-\omega\right)}{\varepsilon\left(q, \xi_{k+q}-\omega\right)}-1\right] \\
& \times\left[\theta\left(w-\xi_{k+q}\right)-\theta\left(-\xi_{k+q}\right)\right] .
\end{aligned}
$$

In these expressions $\Gamma(q, \omega)$ is called the vertex function and we use the local approximation ${ }^{19}$

$$
\Gamma(q, \omega)=\frac{1}{1+V(q) G(q) \chi_{0}(q, \omega)} .
$$

The dielectric function $\varepsilon(q, \omega)$ to be used in the above formulation with the vertex correction is given by $\varepsilon(q, \omega)=1$ $-V(q) \chi_{0}(q, \omega) \Gamma(q, \omega)$, where $\chi_{0}(q, \omega)$ is the densitydensity response function for noninteracting electrons. ${ }^{20}$ The function $G(q)$ is called the local-field correction factor. We employ the $G(q)$ calculated within the Singwi-Tosi-LandSjölander (STLS) approach $^{21}$ by Gold and Calmels. ${ }^{4} \mathrm{We}$ note that the above expressions reduce to the familiar $G W$ approximation forms when the vertex corrections are neglected, i.e., $G(q)=0$ or equivalently $\Gamma(q, \omega)=1$.

In this work, we calculate the self-energy in leading-order perturbation theory. Our expressions for the $G W$ and $G W \Gamma$ self-energies contain noninteracting Green's functions. We have not attempted to perform a self-consistent calculation $^{22,23}$ but surmise that our results would remain qualitatively the same.

\section{RESULTS AND DISCUSSION}

In the numerical calculations to be presented below, we specialize to a quantum wire made out of GaAs material. The material parameters of relevance to us are the dielectric constant $\epsilon_{0}=12.9$ and the electron effective mass $m^{*}$ $=0.07 \mathrm{~m}$. We study the quasiparticle properties for a given density of electrons characterized by $r_{s}$ and lateral quantumwire size $b$. In the examples below, we took $b=a_{B}^{*}$ (which corresponds to $\sim 100 \AA$ for GaAs) but did not perform a systematic study of different wire widths, partly because the local-field correction data ${ }^{4}$ were available only for this value of $b$. However, we believe that our results should be representative, and in principle may be extended to other quantum-wire widths.

In Fig. 1 we show the frequency dependence of the real and imaginary parts of the self-energy calculated in two different approximations: the RPA- $G W$ and the $G W \Gamma$ schemes. The self-energies at $k=k_{F}$ are depicted in Figs. 1(a) and 1(c), for $r_{s}=1$ and $r_{s}=3$, respectively. As expected, the results of the $G W$ approximation are closer to those of the $G W \Gamma$ approximation for higher densities, indicating that vertex corrections are of lesser importance. However, for lower densities (large $r_{s}$ ) there are significant differences between the results of the $G W$ and $G W \Gamma$ approximations. Similar conclusions may be drawn from the self-energy results illustrated in Figs. 1(b) and 1(d), where we consider the band edge $k=0$. The imaginary part $|\operatorname{Im} \Sigma(k, \omega)|$ as a function of $\omega$ has finite discontinuities around $\omega= \pm \omega_{q}\left(k+k_{F}\right)$ within the $G W$ approximation, where $\omega_{q}$ is the plasmon dispersion. $^{20}$ Since $\operatorname{Re}[\Sigma]$ and $\operatorname{Im}[\Sigma]$ are related to each other through the Kramers-Kronig relations, a finite discontinuity in $\operatorname{Im}[\Sigma]$ gives rise to a logarithmic singularity in $\operatorname{Re}[\Sigma]$. In the $G W \Gamma$ calculation of the self-energy, the finite discontinuities in $\operatorname{Im}[\Sigma]$ occur also around $\omega= \pm \omega_{q}(k$ $+k_{F}$ ), where this time $\omega_{q}$ includes the local-field effects. For $r_{s}=1$ and $k=k_{F}, \operatorname{Im}[\Sigma]$ is continuous but its derivative is discontinuous at the same point. In the $G W \Gamma$ approximation calculation of the self-energy, the discontinuities in $\operatorname{Im}[\Sigma]$ occur at larger $|\omega|$ values than $\omega=\left|\omega_{q}\left(k+k_{F}\right)\right|$ for larger $r_{s}$ values. There are considerable differences between the $G W$ and $G W \Gamma$ approximation results. The main reason for this is that with increasing interaction strength the RPA does not provide a good description of the ground-state energy beyond $r_{s} \gtrsim 1$. The local-field corrections restore the quality of approximation in the intermediate coupling region $\left(1 \lesssim r_{s}\right.$ $\lesssim 5$ ), thus the quasiparticle properties calculated within the $G W \Gamma$ approximation are expected to give a better account.

Once the self-energy $\Sigma(k, \omega)$ is known, other quasiparticle properties can be readily evaluated. We now examine the single-particle spectral function $A(k, \omega)$ defined as 

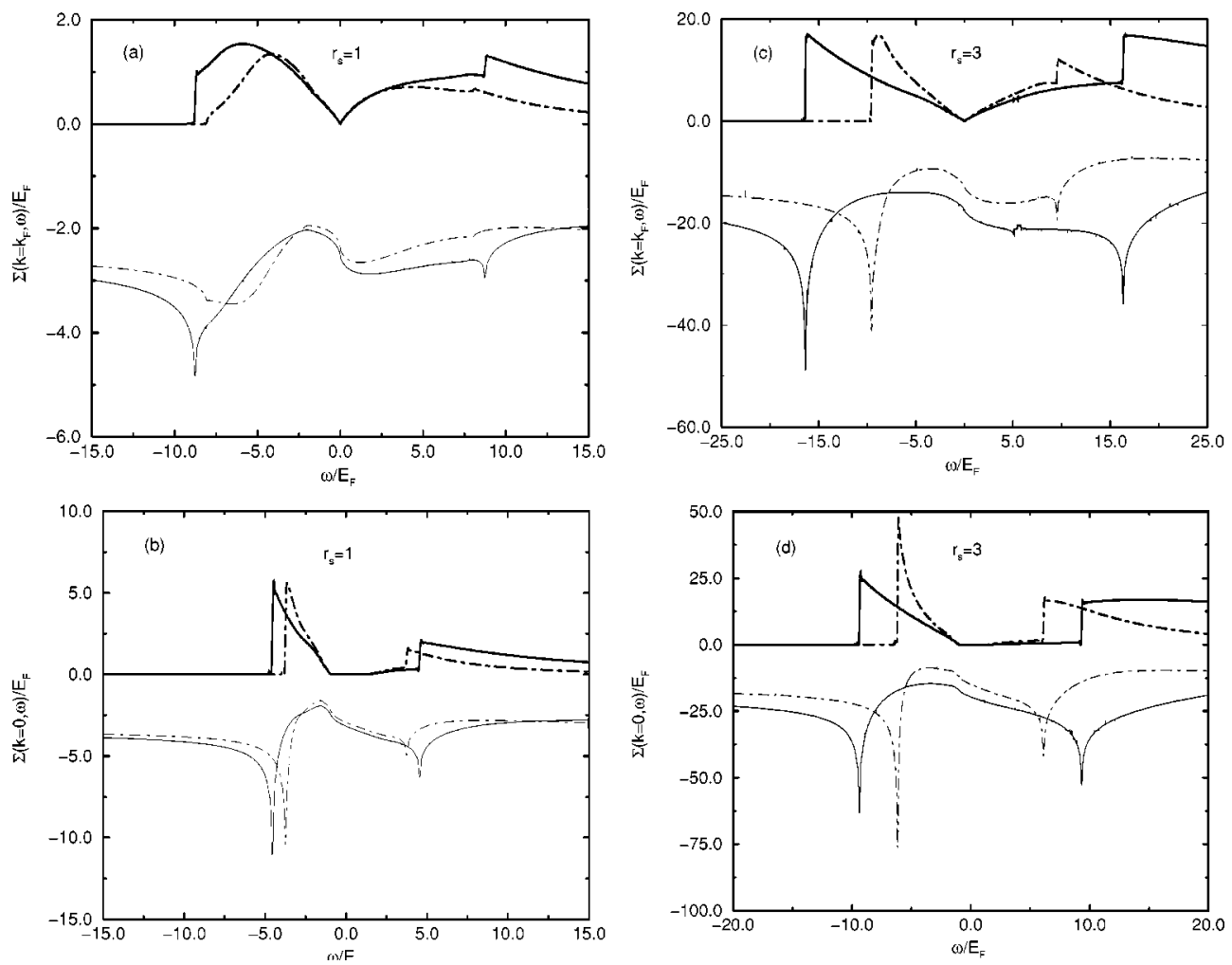

FIG. 1. The self-energy $\Sigma(k, \omega)$ as a function of the frequency $\omega$ for (a) and (b) $r_{s}=1$ and (c) and (d) $r_{s}=3$, for the wave vector values $k=k_{F}$ (a) and (c) and $k=0$ (b) and (d). The solid and dashed lines correspond to the $G W$ (RPA) and $G W \Gamma$ approximations, respectively. The upper and lower set of curves show the $|\operatorname{Im} \Sigma(k, \omega)|$ and $\operatorname{Re} \Sigma(k, \omega)$, respectively.

$$
A(k, \omega)=\frac{2|\operatorname{Im} \Sigma(k, \omega)|}{\left[\omega-\xi_{k}-\operatorname{Re} \Sigma(k, \omega)\right]^{2}+[\operatorname{Im} \Sigma(k, \omega)]^{2}} .
$$

The spectral function $A(k, \omega)$ can be observed experimentally with photoemission spectroscopy, ${ }^{24}$ thus it is important to investigate some of its properties. In Figs. 2(a) and 2(b) we show $A(k, \omega)$ as a function of the frequency at $k=0$ and $k=k_{F}$, respectively, for $r_{s}=1$. We note that the difference between the $G W$ and $G W \Gamma$ approximation results is more prominent at the band edge $k=0$ than at $k=k_{F}$. For $k=0$, there are two sharp peaks in $A(k=0, \omega)$, of which the location of the first one is different in respective approximation schemes. The intersections of $\operatorname{Re}[\Sigma]$ and the straight line $\omega-\xi(k)-\mu$ indicate the solutions to Dyson's equation. For the $k=k_{F}$ case, there is only one solution at $\omega=0$, and therefore, a strong peak in $A(k, \omega)$. This peak is not of a $\delta$-function type, since $\operatorname{Im} \Sigma\left(k_{F}, \omega\right) \rightarrow 0$ as $\omega \rightarrow 0$. At the band edge $(k=0)$, we find three solutions to Dyson's equation, the first one of which corresponds to the regular quasiparticle peak. It is slightly shifted from the noninteracting single-particle energy $\omega=\xi(k)$ and produces a sharp $\delta$-function peak. The second solution has a broad incoherent structure indicating finite damping in the spectral function and does not produce a peak. The third solution is only weakly damped and produces a second sharp $\delta$-function peak in $A(k, \omega)$. This solution has been termed a plasmaron and interpreted as a hole coupled to a cloud of real plasmons. In general, the spectral weight of the plasmaron peak is smaller than the quasiparticle peak, and it produces a satellite peak below the edge of the one-particle density of states, which is experimentally observed in $3 \mathrm{D}$ metallic systems.
We have not carried out a detailed calculation of the plasmaron excitation energies, but in qualitative terms it should be similar to the $2 \mathrm{D}$ case $^{25}$ where plasmarons give rise to a staircaselike structure.

The single-particle spectral density also satisfies the sum rule $\int d \omega /(2 \pi) A(k, \omega)=1$, which we verify numerically to a very high accuracy. The first frequency sum rule yields ${ }^{23}$ $\int d \omega /(2 \pi) \omega A(k, \omega)=E_{k}^{\mathrm{HF}}$, where $E_{k}^{\mathrm{HF}}=\xi_{k}+\Sigma_{\text {ex }}(k)$ is the quasiparticle energy in the Hartree-Fock approximation and the spectral function $A(k, \omega)$ is evaluated in the $G W$ approximation. As an illustration, we compare the right- and left-hand sides of this sum rule in Fig. 3, and find that it is also satisfied within our approximation scheme for the vertex function. The momentum distribution of particles $n(k)$ $=\int_{-\infty}^{0} A(k, \omega) d \omega / 2 \pi$ is shown in Fig. 4 for $r_{s}=1$ and $r_{s}$ $=3$. The expected behavior of a jump discontinuity at $k$ $=k_{F}$ decreases with increasing $r_{s}$, as in a normal Fermi liquid. ${ }^{2}$ We observe that the differences between the $G W$ and $G W \Gamma$ approximations become more significant as $r_{s}$ increases.

The quasiparticle broadening or the damping rate $\Gamma(k)$ is given by the imaginary part of the self-energy,

$$
\Gamma(k)=-\operatorname{Im} \Sigma\left(k, \xi_{k}\right) .
$$

From the damping rate, we can calculate the quasiparticle scattering rate $2 \Gamma(k)$, the inelastic lifetime $\tau(k)$ $=[2 \Gamma(k)]^{-1}$, and the inelastic mean free path $l(k)$ $=v(k) \tau(k)$, where $v(k)$ is the electron velocity. Figure 5 shows $\Gamma(k)$ for $r_{s}=1$ and $r_{s}=3$ in the RPA- $G W$ and $G W \Gamma$ approximations. The damping rate calculated within the 

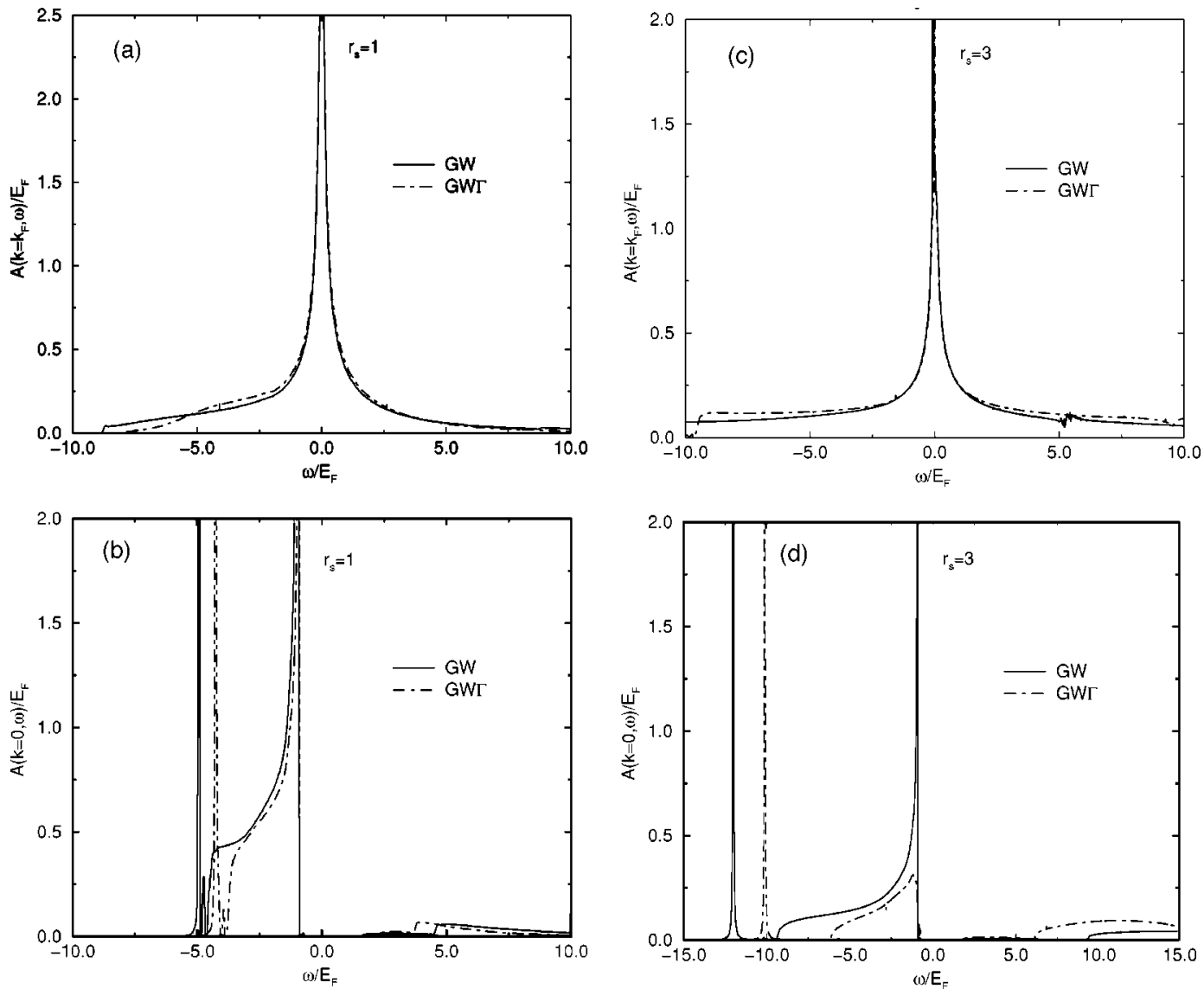

FIG. 2. The single-particle spectral function $A(k, \omega)$ as a function of frequency for (a) and (b) $r_{S}=1$ and (c) and (d) $r_{s}=3$, for the wave vector values $k=k_{F}$ (a) and (c) and $k=0$ (b) and (d).

$G W \Gamma$ approximation is not drastically different in its overall shape than the one calculated within the $G W(\mathrm{RPA})$. However, $\Gamma(k)$ within the $G W \Gamma$ is in general smaller in magnitude than that in the RPA- $G W$. This is consistent with the earlier results shown in Fig. 1. A similar comparison for 2D electron systems was made by Marmorkos and Das Sarma. ${ }^{14}$ There, it was found that the vertex corrections were only 10-30\% smaller than the RPA results. The large decrease in $\Gamma(k)$ (especially at larger $r_{s}$ values) may be partly due to the dimensionality and partly due to the relative strength of the interaction. In any case, our results indicate the significance of vertex corrections or correlation effects beyond the RPA. For wave vectors away from $k_{F}$, but less than some threshold wave vector $k_{c}\left(k_{c} \approx 2.7 k_{F}\right.$ for $r_{s}=1$ and $k_{c} \approx 4.9 k_{F}$ for $r_{s}=3$ in the RPA), damping rates including the vertex corrections are higher than those in the RPA. In the large wave-vector regime, indicated by the sharp increase in $\Gamma(k)$ in Fig. 5, the plasmon excitation mechanism becomes important. Here the effects of vertex corrections are observed to decrease the damping rate. Furthermore, the threshold wave vector $k_{c}$ for the onset of plasmons decreases compared to the RPA values $\left(k_{c} \approx 2.3 k_{F}\right.$ for $r_{s}=1$ and $k_{c} \approx 3.1 k_{F}$ for $r_{s}=3$ in the $G W \Gamma$ approximation). As is known, the vertex corrections (local-field corrections) in general lower the plasmon energies. ${ }^{3-6}$ As argued before, for larger values of $r_{s}$ the RPA breaks down, and the results which include the localfield corrections (i.e., $G W \Gamma$ approximation) should be more trustworthy.

The above examples of quasiparticle properties suggest that as far as the Fermi liquid concept is applicable, the vertex corrections are significant at large $r_{s}$ values. Since the various ground-state energy calculations and quantum Monte Carlo simulations suggest the inclusion of correlation effects beyond the RPA for $r_{s} \gtrsim 1$, our investigation of the quasiparticle properties provides a consistent picture with the above framework. Various results presented in this work may in principle be compared to the experiments when they become available. Our vertex function $\Gamma(q, \omega)$ is limited by the local-field corrections obtained within the self-consistent-

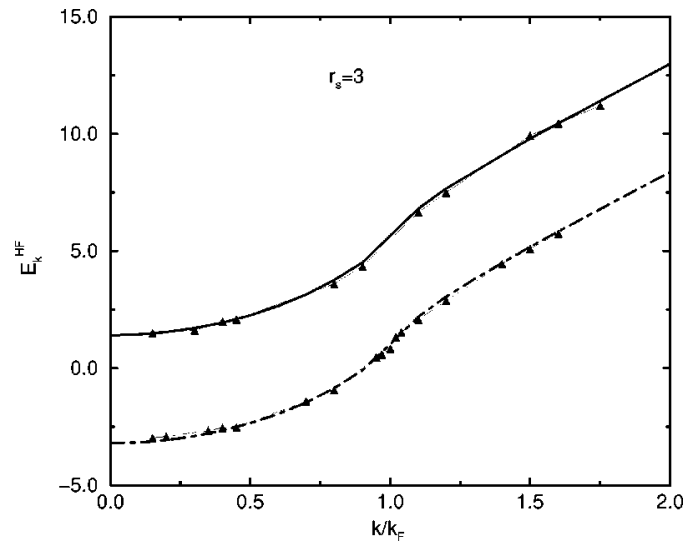

FIG. 3. The Hartree-Fock energy $E_{k}^{\mathrm{HF}}$ for within the $G W$ (upper curves) and $G W \Gamma$ (lower curves) schemes at $r_{s}=3$. The results from the first moment of $A(k, \omega)$ are indicated by triangles which are joined by thin lines to guide the eye. 


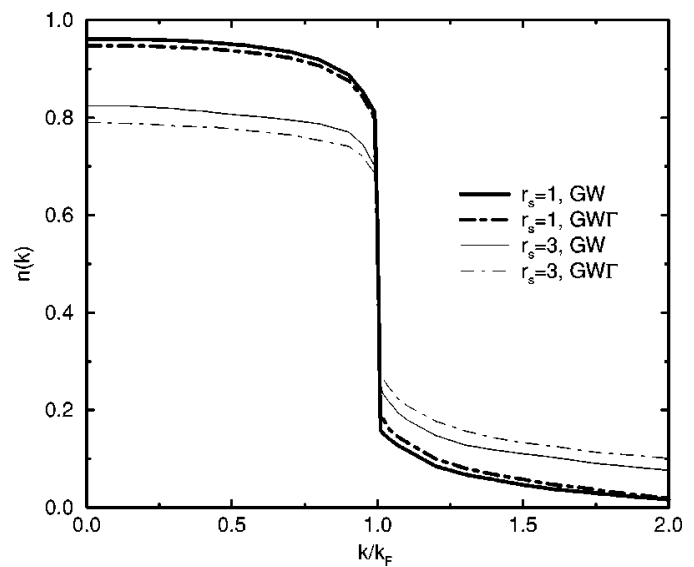

FIG. 4. The momentum distribution $n(k)$ of interacting electrons within the $G W$ and $G W \Gamma$ schemes at $r_{s}=1$ and $r_{s}=3$.

field scheme. Better approximations to the $G(q)$, in particular those satisfying the exact limiting forms and various sum rules, may be constructed for improvement. ${ }^{13}$ The frequency dependence of $\Gamma(q, \omega)$ may be improved at the STLS approximation level by considering a dynamical local-field factor $^{7} G(q, \omega)$. It would be interesting to use $G(q, \omega)$ when constructing $\Gamma(q, \omega)$ and study its effects on the quasiparticle properties. Such a calculation is beyond the scope of the present study, but we can guess that dynamical effects should somewhat modify the frequency dependence of the self-energy and the spectral function. More specifically, the location of singularities in $\Sigma(k, \omega)$ will be shifted, since the plasmon frequency $\omega_{q}$ will be different in the dynamical approach.

For quantum wires with long-range Coulomb interaction, Das Sarma, Hwang, and Zheng ${ }^{8}$ developed a plasmon-pole approximation which turns out to be very accurate in the calculation of quasiparticle properties. Here, we generalize their account to include the local-field effects. We first note that in the $G W \Gamma$ approximation when a local-field factor $G(q)$ is used in the description of the vertex function $\Gamma(q, \omega)$, the various cancelations render the self-energy expression the same as in the $G W$ approximation, except that the screening function becomes $\varepsilon(q, \omega)=1-V(q)[1$ $-G(q)] \chi_{0}(q, \omega)$. Using the plasmon-pole approximation ${ }^{11}$ for the density-density correlation function $\chi_{0}(q, \omega)$, we obtain

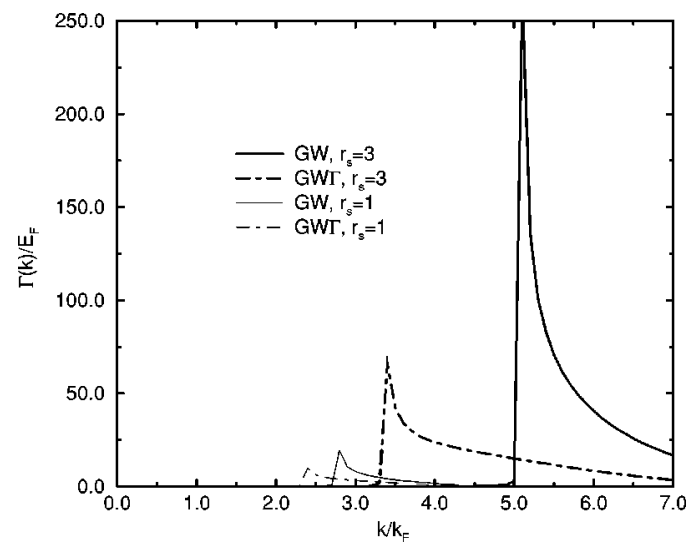

FIG. 5. The damping rate $\Gamma(k)$ as a function of $k$ for $r_{s}=1$ and $r_{s}=3$ in the $G W$ and $G W \Gamma$ approximations.

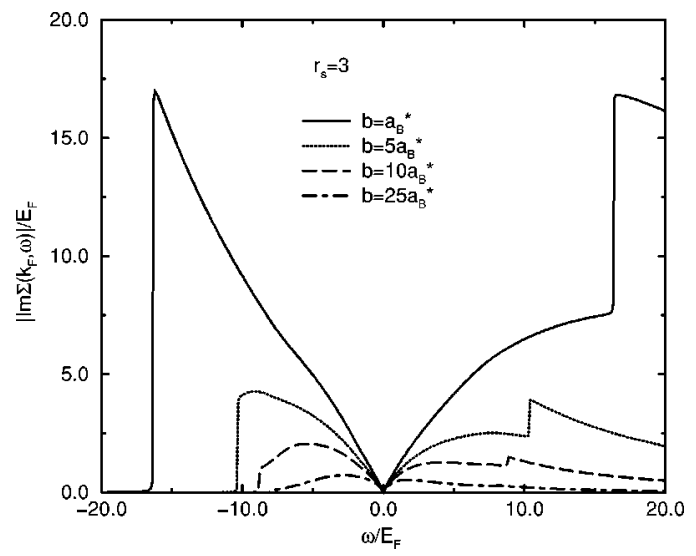

FIG. 6. The imaginary part of the self-energy at the Fermi surface $\left|\operatorname{Im} \Sigma\left(k_{F}, \omega\right)\right|$ as a function of $\omega$, for various values of the quantum-wire width parameter.

$$
\varepsilon(q, \omega)=1-\frac{[1-G(q)] \omega_{0}}{\omega^{2}-\omega_{q}+\omega_{0}^{2}+i \eta}
$$

where the pole strength $\omega_{0}^{2}=(n / m) V(q) q^{2}$ is determined by the $f$-sum rule and $\omega_{q}$ is the $1 \mathrm{D}$ plasmon dispersion. ${ }^{20}$ In the above form of $\varepsilon(q, \omega)$, the RPA is recovered when $G(q)$ $=0$. With these modifications, the calculation of the selfenergy within the plasmon-pole approximation (including the vertex corrections) proceeds along the lines given by Das Sarma, Hwang, and Zheng. ${ }^{8}$ We have selectively tested the plasmon-pole approximation in the presence of vertex corrections and found reasonable agreement with the fully numerical results. The level of agreement is essentially the same as that obtained by Das Sarma, Hwang, and Zheng ${ }^{8}$ for the $G W$ approximation. Finally, we mention that our calculations including the vertex corrections may be extended to include the phonon effects to describe the quantum wires made of weakly polar materials (such as GaAs) similarly to the previous related works. ${ }^{26}$

As mentioned before, our self-energy calculations are performed for the quantum-wire width of $b=a_{B}^{*}$ only, because of the availability of the local-field-factor data. It would be of interest to systematically study the dependence of the quasiparticle properties on increasing $b$, to observe the changes from a Q1D behavior to that of 2D. There are definite predictions given by $\mathrm{Hu}$ and Das Sarma, ${ }^{2}$ for instance, for the behavior of $\left|\operatorname{Im} \Sigma\left(k_{F}, \omega\right)\right|$ as $\omega \rightarrow 0$, due to the plasmon and single-particle contributions in Q1D and 2D systems. Although the Coulomb interaction $V(q)$ $=\left(e^{2} / \epsilon_{0}\right) e^{(b q / 2)^{2}} K_{0}\left[(b q / 2)^{2}\right]$ approaches $\sim 1 / q$ for large $b$ as in 2D systems, it is not clear that the dielectric function $\varepsilon(q, \omega)$ which describes the plasmon and single-particle excitations would display a 2D-like behavior. With the asymptotic form of $V(q)$ and 1D dielectric function, we went through the analysis of $\mathrm{Hu}$ and Das Sarma ${ }^{2}$ to roughly find $\left|\operatorname{Im} \Sigma\left(k_{F}, \omega\right)\right| \sim \omega^{2 / 3}$ as $\omega \rightarrow 0$, which is different from the limit predicted for a pure $2 \mathrm{D}$ calculation, viz., $\sim \omega^{2}$. It appears that a crossover behavior within the present model may not be observed. More detailed calculations would be required to determine the precise conditions and search for a crossover behavior. For illustration purposes, we show in Fig. $6\left|\operatorname{Im} \Sigma\left(k_{F}, \omega\right)\right|$ as a function of $\omega$, for various values of 
the width parameter within the RPA (i.e., the $G W$ approximation). We do not find any conclusive evidence for a crossover behavior in our sketchy analysis.

To summarize, we have calculated the quasiparticle properties of a 1D electron gas interacting with a long-range repulsive Coulomb potential. We have used the RPA-based $G W$ approximation and the $G W \Gamma$ approximation, which includes the local-field corrections to calculate the electron self-energy. We found that a number of quasiparticle properties are strongly affected by the inclusion of vertex corrections as the electron density is decreased. The plasmon-pole approximation to the dielectric function in the self-energy calculations including the vertex corrections seems to agree with the full $G W \Gamma$ approximation, which may be utilized in more demanding calculations.

\section{ACKNOWLEDGMENTS}

This work was partially supported by the Scientific and Technical Research Council of Turkey (TUBITAK) (TBAG1662), by NATO (SfP971970), and by the Turkish Department of Defense (KOBRA-001).
${ }^{1}$ D.C. Mattis, The Many Body Problem (World Scientific, Singapore, 1993).

${ }^{2}$ B.Y-K. Hu and S. Das Sarma, Phys. Rev. B 48, 5469 (1993).

${ }^{3}$ W.I. Friesen and B. Bergersen, J. Phys. C 13, 6627 (1980).

${ }^{4}$ A. Gold and L. Calmels, Solid State Commun. 100, 137 (1996).

${ }^{5}$ J.S. Thakur and D. Neilson, Phys. Rev. B 56, 4679 (1997).

${ }^{6}$ D. Agosti, F. Pederiva, E. Lipparini, and K. Takayanagi, Phys. Rev. B 57, 14869 (1998).

${ }^{7}$ B. Tanatar and C. Bulutay, Phys. Rev. B 59, 15019 (1999).

${ }^{8}$ S. Das Sarma, E.H. Hwang, and L. Zheng, Phys. Rev. B 54, 8057 (1996).

${ }^{9}$ I.E. Dzyaloshinskii and A.I. Larkin, Zh. Éksp. Teor. Fiz. 65, 411 (1973) [JETP Lett. 38, 202 (1974)].

${ }^{10}$ A.R. Goni, A. Pinczuk, J.S. Weiner, J.M. Calleja, B.S. Dennis, L.N. Pfeiffer, and K.W. West, Phys. Rev. Lett. 67, 3298 (1991).

${ }^{11}$ Q.P. Li, S. Das Sarma, and R. Joynt, Phys. Rev. B 45, 13713 (1992).

${ }^{12}$ A. Malatesta and G. Senatore, J. Phys. IV 10, 341 (2000); A. Malatesta, Ph. D. thesis, University of Trieste (1999) (unpublished).

${ }^{13}$ G.E. Santoro and G.F. Giuliani, Phys. Rev. B 39, 12818 (1989).

${ }^{14}$ I.K. Marmorkos and S. Das Sarma, Phys. Rev. B 44, 3451 (1991).

${ }^{15}$ G.Y. Hu and R.F. O'Connell, Phys. Rev. B 42, 1290 (1990).

${ }^{16}$ J.J. Quinn and R.A. Ferrell, Phys. Rev. 112, 812 (1958); G. D.
Mahan, Many Particle Physics (Plenum, New York, 1990).

${ }^{17}$ L. Hedin, Phys. Rev. 139, 796 (1965). For a recent review of the method and applications, see, for instance, F. Aryasetiawan and O. Gunnarsson, Rep. Prog. Phys. 61, 237 (1998); L. Hedin, J. Phys.: Condens. Matter 11, 489 (1999).

${ }^{18}$ See, for instance, A. Schindlmayr and R.W. Godby, Phys. Rev. Lett. 80, 1702 (1998); A. Virosztek and J. Ruvalds, Phys. Rev. B 59, 1324 (1999).

${ }^{19}$ G.D. Mahan and B.E. Sernelius, Phys. Rev. Lett. 62, 2718 (1989).

${ }^{20}$ P.F. Williams and A.N. Bloch, Phys. Rev. B 10, 1097 (1974).

${ }^{21}$ K.S. Singwi, M.P. Tosi, R.H. Land, and A. Sjölander, Phys. Rev. 176, 589 (1968)

${ }^{22}$ H.J. de Groot, P.A. Bobbert, and W. van Haeringen, Phys. Rev. B 52, 11000 (1995); S. Schäfer and P. Schuck, ibid. 59, 1712 (1999).

${ }^{23}$ U. von Barth and B. Holm, Phys. Rev. B 54, 8411 (1996).

${ }^{24}$ C. Kim, A.Y. Matsuura, Z.-X. Shen, N. Motoyama, H. Eisaki, S. Uchida, T. Tohyama, and S. Maekawa, Phys. Rev. Lett. 77, 4054 (1996).

${ }^{25}$ P. von Allmen, Phys. Rev. B 46, 13345 (1992).

${ }^{26}$ E.H. Hwang, B. Y.-K. Hu, and S. Das Sarma, Phys. Rev. B 54, 4996 (1996); R. Jalabert and S. Das Sarma, ibid. 40, 9723 (1989). 